\title{
Identification of cell types in multiplexed in situ images by combining protein expression and spatial information using CELESTA reveals novel spatial biology
}

Weiruo Zhang ${ }^{1,2}$, Irene $\mathrm{Li}^{1,2,3}$, Nathan E. Reticker-Flynn ${ }^{4}$, Zinaida Good ${ }^{1,2,5}$, Serena Chang ${ }^{5,6}$, Nikolay Samusik ${ }^{4}$, Saumyaa Saumyaa ${ }^{5,6}$, Yuanyuan $\mathrm{Li}^{1,2}$, Xin Zhou ${ }^{7}$, Rachel Liang $^{8}$, Christina S. Kong ${ }^{4,5}$, Quynh-Thu Le ${ }^{8}$, Andrew J. Gentles ${ }^{1,6,9}$, John B. Sunwoo ${ }^{5,6}$, Garry P. Nolan ${ }^{4}$, Edgar G. Engleman ${ }^{4}$, Sylvia K. Plevritis*1,2

${ }^{1}$ Department of Biomedical Data Science, School of Medicine, Stanford University, CA, USA

${ }^{2}$ Department of Radiology, School of Medicine, Stanford University, CA, USA

${ }^{3}$ Cancer Biology Program, School of Medicine, Stanford University, CA, USA

${ }^{4}$ Department of Pathology, School of Medicine, Stanford University, CA, USA

${ }^{5}$ Stanford Cancer Institute, Stanford University, CA, USA

${ }^{6}$ Division of Head and Neck Surgery, Department of Otolaryngology, School of Medicine, Stanford University, CA, USA

${ }^{7}$ Department of Computer Science, Stanford University, CA, USA

${ }^{8}$ Department of Radiation Oncology, School of Medicine, Stanford University, CA, USA

${ }^{9}$ Department of Medicine, Stanford University, CA, USA

* corresponding author

Contact information:

Sylvia Plevritis: plevriti@stanford.edu

\section{ABSTRACT}

Advances in multiplexed in situ imaging are revealing important insights in spatial

biology. However, cell type identification remains a major challenge in imaging analysis, with most existing methods involving substantial manual assessment and subjective

31 CELESTA, which uses both cell's protein expression and spatial information to identify cell type of individual cells. We demonstrate the performance of CELESTA on multiplexed immunofluorescence in situ images of colorectal cancer and head and neck

34 cancer. Using the cell types identified by CELESTA, we identify tissue architecture associated with lymph node metastasis in HNSCC, which we validate in an independent 
36 cohort. By coupling our in situ spatial analysis with single-cell RNA-sequencing data on

37 proximal sections of the same tissue specimens, we identify and validate cell-cell

38 crosstalk associated with lymph node metastasis, demonstrating the power of spatial

39 biology to reveal clinically-relevant cellular interactions.

\section{INTRODUCTION}

42 Spatial biology is a new frontier that provides unprecedented characterization of tissue

43 architectures through technological advances in multiplexed in situ imaging platforms ${ }^{1-6}$.

44 Using these platforms, specific tissue architectures have been associated with tissue

45 development, disease progression and treatment response ${ }^{7-10}$. However, such insights

46 often rely on substantial data preprocessing that can introduce biases. In the case of $i n$

47 situ image, raw pixel data often need to be converted into images of cells through cell

48 segmentation followed by identification of cell types for individual cells. Current cell

49 type identification methods typically involves manual gating and/or clustering cells based

50 on their protein expression profiles. Moreover, manual gating is subjective ${ }^{11,12}$ and

51 becomes unmanageable with high dimensional data. Clustering cells with similar protein

52 expressions can be biased through the selection of number of clusters and it is often the

53 case that individual clusters are a mixture of cell types, thereby comprising the single-cell

54 resolution of the data ${ }^{13}$. Even after clustering, decisions to assign cell types to the

55 clusters are subjective, and clusters with cell type mixtures particularly demand much

56 manual assessment. Given these limitations, downstream analysis of cellular spatial

57 patterns can be highly compromised. 
58 To address the limitations of common cell type identification methods, we reason that

59 cells are organized in coherent spatial patterns and a cell's spatial neighborhood is

60 valuable information and could be considered together with cell's protein expression

61 profile to infer its cell type. We propose a novel machine learning cell type identification

62 method, termed "CELESTA" (CELl typE identification with SpaTiAl information), that

63 incorporates both cell's protein expression profile and its spatial information, with

64 minimal to no user-dependence, to produce relatively fast cell type assignments.

65 CELESTA assigns cells to their most likely cell types through an optimization framework

66 based on Markov Random Field modeling and relies on an input of prior information on

67 cell type markers, thereby leveraging biological knowledge in a non-subjective manner.

69 We demonstrate the performance of CELESTA on imaging data generated using the

70 CODEX (CO-Detection by indEXing) platform ${ }^{14}$. CODEX is an immunofluorescence-

71 based multiplexed in situ imaging technology that quantifies a cell's spatial location and

72 expressions of over fifty proteins, across tens of thousands of cells of a tissue slice ${ }^{14}$. We

73 applied CELESTA to a published CODEX dataset generated on colorectal cancer where

74 cell type identification is based on both unsupervised and supervised clustering and

75 manual assessment by pathologists ${ }^{6}$, which we adopt as the gold standard for

76 benchmarking CELESTA. CELESTA provides cell type assignments comparable to the

77 gold standard. Moreover, CELESTA provides assignments to cells grouped in clusters

78 which were left as unassessed through manual annotation because the clusters were

79 regarded a mixture of different cell types. Since CELESTA does not rely on clustering, it

80 was able to identify these unlabeled cell types. 
82 To identify tissue architectures associated with lymph node metastasis, we applied

83 CELESTA on our study cohort of head and neck squamous cell carcinoma (HNSCC)

84 where we generated CODEX images from patients with (N+) and without (N0) lymph

85 node metastasis. We identified specific cell types that were hypothetically colocalized

86 only in N+ HNSCC patients and validated our findings on an external HNSCC cohort of

87 tissue microarray (TMA). By coupling our spatial analysis with single-cell RNA-

88 sequencing data on proximal sections of the same tissue specimens of the imaging data,

89 we identified and validated cell-cell crosstalk associated with lymph node metastasis,

90 demonstrating the power of spatial biology to reveal clinically-relevant cell-cell

91 interactions.

92

93 RESULTS

94 Analysis Overview. Our analysis pipeline was designed to associate tissue architecture in 95 a primary tumor with the nodal status (Figure 1a). Our study started with multiplexed in

96 situ images on which we performed segmenting pixel-based data into cells, and identified

97 cell types for each segmented cell using CELESTA. We then performed spatial analysis

98 to characterize the tissue architecture to identify statistically-significant cell-cell

99 colocalization patterns associated with nodal status and generate spatial biological

100 hypothesis. By coupling this spatial biology with scRNA-seq analysis, we identified

101 potential cell-cell crosstalk associated with lymph node metastasis.

102

103 Overview of CELESTA. CELESTA is an unsupervised machine learning cell type 
104 identification method. CELESTA is currently optimized for in situ multiplexed

105 immunofluorescence-based imaging but has broader applicability. Post cell segmentation,

106 CELESTA first assigns cell types to cells whose protein expression profiles clearly match

107 prior knowledge of cell-type-specific markers; those cells are defined as "anchor cells."

108 For the remaining cells, whose protein expression profile does not have clear pattern

109 associated with a cell type, referred to hereon as "index cells", CELESTA uses the

110 neighboring cell-type information in addition to the cell's protein expression profile to

111 identify the cell type. Cell types for index cells are assigned through an iterative

112 optimization framework. Illustration of CELESTA cell type assignment in the tissue is

113 provided in Figure 1b and 1c. A flowchart illustrating CELESTA algorithm is shown in

$114 \quad$ Figure 2a.

115

116 CELESTA requires two inputs. The first input is an in situ image that has been

117 segmented into individual cells where each cell is defined by its protein expression

118 profile and spatial location, described in terms of $\mathrm{X}$ and $\mathrm{Y}$ coordinates (Figure 2b). In a

119 preprocessing step, CELESTA defines the "cell neighborhood" using N-nearest

120 neighbors based on cell's spatial X and Y coordinates (Figure 2b). CELESTA then

121 determines whether or not a protein marker is overexpressed in a given cell relative to

122 other cells by fitting a two-mode Gaussian mixture model to the protein expressions

123 across all the cells in a given sample ${ }^{15}$; this step identifies the distributions for high vs.

124 low expression levels. CELESTA then converts the protein expression into an

125 "expression probability" based on a sigmoid function where the midpoint is the

126 intersection of posterior probabilities based on the two-mode Gaussian distribution 
127 (Figure 2b). After preprocessing, each protein expression is scaled between 0 and 1 as a

$128 \quad$ probability.

130 The second input to CELESTA is a cell-type signature matrix that defines each cell type

131 in terms of its protein signature. This signature matrix relies on prior knowledge of

132 proteins known to be expressed in specific cell types, given protein markers in the

133 imaging panel (Figure 2b). For each cell type, the cell-type signature matrix indicates

134 whether a protein is expressed in that cell type, initialized as 1 if expressed or 0 if not

135 expressed, and updated with cells assigned. If the expression of a specific protein is

136 irrelevant to identity the cell type, the protein is denoted as "NA" for that cell type. An

137 example initial cell-type signature matrix on the public colorectal cancer dataset is shown

138 in Table S1.

$140 \quad$ For cell type assignment, CELESTA first matches a cell's expression probabilities to

141 cell-type signatures to determine if it is sufficient to identify its cell-type (Figure 2a(i)).

142 To make the assessment, CELESTA relies on a scoring function (Figure 2a(i), 2c),

143 defined as 1 minus the mean squared errors between expression probabilities of a cell and

144 the reference protein expression profile for each cell type in the initial cell-type signature

145 matrix (Figure 2c). When a cell has one dominate cell type score, CELESTA assigns the

146 corresponding cell type to that cell and defines the cell as an "anchor cell" (Figure

147 2a(ii)). CELESTA then updates the cells' neighborhood cell types and cell-type

148 signature matrix (Figure 2a(iii)) using the information from anchor cells. 
$150 \quad$ For a cell whose cell type cannot be identified using protein expression alone (index cell,

151 Figure 2a(iv)), CELESTA defines an energy function (Figures 2a(v), 2d) using the Potts

$152 \operatorname{model}^{16}$ to leverage cell type information on its spatially N-nearest neighboring cells in a

153 probabilistic manner. The Potts model has been used for image segmentation where an

154 indicator function is applied to increase the potential of the neighboring pixels belonging

155 to the same object ${ }^{17-19}$. The Potts model has also been demonstrated useful to analyze

156 pathological images ${ }^{20}$. CELESTA extends the concept of Potts model to spatial

157 neighborhoods of cells, such that if there is one dominant cell type among the

158 neighboring cells, that cell type contributes higher potential. Incorporating both energy

159 function (Figure 2a(v)) and scoring function (Figure 2a(vi)), CELESTA represents each

160 index cell as a node in an undirected graph with each edge connecting its spatially N-

161 nearest neighbors. CELESTA associates each node with a hidden state, where the hidden

162 state is the cell type to be inferred, and assumes that the joint distribution of the hidden

163 states satisfy discrete Markov Random Field (Figure 2a(vii), 2e). To maximize the joint

164 probability objective function, CELESTA employs an EM algorithm using mean field

165 approximation ${ }^{21}$. In each iteration, cell types with maximum clear-cut probabilities are

166 assigned to the index cells (Figure 2a(viii)). The neighborhood cell types and signature

167 matrix are also updated in each iteration (Figure 2a(ix)). If the protein expression profile

168 together with the spatial information still does not produce clear-cut on probability for a

169 cell type, CELESTA re-evaluates the cell on the next iteration with more neighboring

170 cells assigned cell types (Figure $\mathbf{2 a}(\mathbf{x}))$. The process is repeated until remaining number

171 of cells is smaller than a user defined convergence limit. The cells without cell type

172 identities are assigned to an "unknown" category. 
$174 \quad$ Incorporating cell lineage information into cell type identification. Because assigning

175 cell types to tens of thousands cells is computationally expensive, CELESTA introduces

176 a "cell-type resolution" strategy, whereby the cell type assignment is performed in

177 multiple rounds where each round introduces increasing cell-type resolution based on

178 known cell lineages (Figure 2f). This approach not only reduces computational

179 complexity but also improves robustness when cell types from different lineages have

180 shared marker expression. More details on CELESTA are provided in Methods.

181

182 Comparison of CELESTA with published annotations. We assessed the performance of

183 CELESTA on a public domain CODEX dataset where we used the published cell types as

184 "ground truth." This dataset is a TMA composed 70 cores of human colorectal cancers. In

185 the published study, the cell types were identified by applying both unsupervised ${ }^{22}$ and

186 supervised clustering together with pathologists' manual assessment of marker

187 expressions, cell morphology and staining colocalization ${ }^{6}$. The CELESTA assignments,

188 which were automated and computed on the order of minutes, were consistent with expert

189 assessment. A comparison of CELESTA and published annotations on a representative

190 TMA core is shown in Figure 3a. The number of cells identified per TMA core for each

191 cell type were highly correlated between CELESTA and published annotations (Figure

192 3b,c). Using the published cell type assignments as the gold-standard, we showed that

193 CELESTA achieved an average accuracy score (Rand Index) of around 0.9 across the cell

194 types (Figure 3d); CELESTA has average precision scores ranged between 0.6 to 0.8 and

195 F1 scores ranged between 0.6 to 0.7 across the major cell types (Figure 3d). For rare 
populations that are hard to identify, CELESTA achieved average precision and F1 scores ranged between 0.4 to 0.6 . Noteworthy, there are two clusters in the published

198 annotations that were assigned as cell type mixtures (Figure 3e, f); for cells in these two

199 clusters, CELESTA assigned cell types matched with canonical marker expression

200 patterns (Figure 3e, f), demonstrating CELESTA's unique ability to provide cell type

201 identification at single-cell resolution, not relying on clustering.

203 To evaluate the mismatched cell type assignments between CELESTA and published

204 assignments, we evaluated the protein expression profiles. For example, among the tumor

205 cells that we mismatched, we analyzed the protein expressions of the tumor cells

206 identified by: (i) both methods, (ii) only in the published annotations and (iii) only by

207 CELESTA (Figure S1). Tumor cells that were identified the published annotations but 208 not by CELESTA express very low to none cytokeratin, which is a common tumor cell 209 marker. Tumor cells identified by CELESTA express cytokeratin as defined in the input 210 of cell-type signature matrix.

212 CELESTA applied to primary HNSCC tumors imaged by CODEX. We generated a

213 study cohort of eight primary HNSCC tumors, comprised of four node-positive $(\mathrm{N}+)$ and

214 four node-negative (N0) samples (Table S2). We performed CODEX imaging using the

215 protein marker panel in Table S3. The input cell-type signature matrix based on the

216 imaging panel is shown in Table S4. We manually assessed CELESTA's performance by

217 mapping CELESTA assigned cell types onto the CODEX images using the $\mathrm{X}$ and $\mathrm{Y}$

218 coordinates against the canonical marker staining (Methods). We showed qualitatively 
that CELESTA assigned cell types matched well with canonical marker staining (Figure 4a, 4b, S2). Next, we evaluated cell type composition from CELESTA with paired

221 scRNA-seq data on proximal tissue sections from four samples in our HNSCC cohort

222 (two N+ and two N0 samples). CELESTA identified cell type compositions were

223 correlated with scRNA-seq cell type compositions $(\mathrm{R}=0.7, \mathrm{P}<0.0001$, Figure 4c). While

224 the cell type compositions are concordance, the differences may arise because tissue

225 dissociation methods used for generating scRNA-seq data often over-enriched for

226 immune cells.

227

228 To further quantitatively evaluate the performance of CELESTA, we applied manual

229 gating as benchmark. Due to the limitation of gating on high dimensionality data, we

230 designed gating strategies focusing on cell types relevant with downstream analysis

231 (Methods and Figure S3). Compared with manual gating, CELESTA achieved sample

232 adjusted rand index (ARI) ranged from 0.6 to 0.9 across the samples (Figure 4d). Due to

233 imaging artifacts, cell type identification was harder in some tissue samples. In terms of

234 cell type compositions, CELESTA and manual gating were highly correlated $(\mathrm{R}=0.8$,

$235 \quad \mathrm{p}<0.0001$, Figure 4e). For cell-type specific assessment, CELESTA achieved average F1

236 scores of around 0.7 and average accuracy scores of around 0.9 for malignant cells,

237 endothelial cells and T cells (Figure 4f). For T cell subtypes, CELESTA achieved

238 average F1 scores around 0.55 and average accuracy scores around 0.9 compared with

239 manual gating (Figure 4f). Because T cell subtypes were identified in later rounds of

240 manual gating sequence, potential accrual manual errors could cause reduced accuracy

241 measures. 
244 in primary HNSCC dependent on nodal status. We performed spatial analysis on our

245 HSNCC study cohort using the cell types identified by CELESTA. We adapted the co-

246 location quotient ${ }^{23}$ (CLQ), a measurement used in geospatial statistics, to quantify spatial

247 co-localization between pairs of cell types and test the hypothesis that there are

248 differential cell-type-specific co-localization patterns in N+ vs. N0 HNSCC (Figure 5a).

249 Specifically, by denoting cell-type $a$ as target cells and cell-type $b$ as neighboring cells,

250 we used CLQ to measure the degree to which cell-type $b$ is spatially co-localized with

251 cell-type $a$ and calculated the CLQs for each pair-wise cell types for each sample and

252 compared the CLQs between N+ and N0 samples. Four pairs of cell types that were

253 significantly more co-localized in N+ vs. N0 samples (Figure 5b) (p-values $<0.05)$,

254 namely: (i) Malignant cells and Treg cells, (ii) CD4+ T cells and endothelial cells, (iii)

255 CD8 $+\mathrm{T}$ cells and CD4+ T cells, and (iv) CD4+ T cells with themselves. The hypothetical

256 spatial co-localization pattern differences between $\mathrm{N} 0$ and $\mathrm{N}+$ samples are illustrated in

257 Figure 5c. Representative pairs of CODEX images show FOXP3 (a canonical Treg

258 marker) expression is more co-localized with cytokeratin (tumor marker) expression in

259 N+ vs. N0 HNSCC (Figure 5d), and CD4 and CD8 (canonical T cell markers)

260 expression are more co-localized with CD31 (a canonical endothelial marker) expression

261 in N+ vs. N0 HNSCC (Figure 5e). To validate the hypothesis of co-localization of Tregs

262 and malignant cells in N+ HNSCC, we stained FOXP3 and cytokeratin on a TMA from

263 an independent cohort of 76 HNSCC patients. Representative TMA images illustrating

264 more co-localization of cytokeratin-positive malignant cells and Tregs in N+ vs. N0 
samples are provided in Figure 5f. We showed that quantitatively primary N+ HNSCC Figure 5g) using a TMA-image processing pipeline (Methods). architecture associated with lymph node metastasis in HNSCC. By reasoning that data that was generated on tissue specimens proximal to the imaged specimens (Figure vs. N0 HNSCC (Figure 6b, 6c). We also identified a T cell cluster (Cluster 2) as Trega receptor of CXCL10, was more expressed on N+ vs. N0 HNSCC in the Treg cluster (Figure 6c). This finding led us to hypothesize the CXCL10-CXCR3 interaction as an axis for crosstalk between malignant cells and Tregs in N+ HNSCC. Evidence for this CXCL10-CXCR3 interaction was found in an independent public scRNA-seq HNSCC dataset ${ }^{28}$ (Figure S6). In a similar manner, we found that the ligand-receptor pair

284 CCL20-CCR6 is higher in N+ vs. N0, and may mediate crosstalk between endothelial 285 and CD4+ T cells in N+ HNSCC (Figure 6d). In summary, we demonstrate that 286 CELESTA enabled cell-cell co-localization patterns that guided scRNA-seq analysis to 287 identify cell-cell crosstalk mediators related to lymph node metastasis (Figure 6e). 


\section{Functional validation on CXCL10-CXCR3 crosstalk between malignant cells and}

Tregs. To validate the association of CXCL10-CXCR3 between malignant cells and

291 Tregs with nodal status, we leveraged an in vivo model of lymph node (LN) metastasis ${ }^{29}$

292 that we developed in melanoma. In this model, we created multiple generations of lymph

293 node metastatic cell lines (LN1-LN6), with each generation exhibiting an increased

294 frequency of lymph node metastases. RNA sequencing of different LN lines revealed that

295 later generations (LN6) expressed significantly higher levels of CXCL10 compared with

296 the parental cell line (Figure 6f), indicating metastatic cells to the lymph node upregulate

297 CXCL10.

299 We tested the hypothesis that CXCR3+ Tregs are more attracted to CXCL10+ malignant

300 cells in a transwell experiment. Tregs harvested from naive FOXP3-GFP mice were

301 plated in the upper chambers; the bottom chambers were plated with either the parental

302 melanoma cells (control group) or LN6 melanoma cells (study group). We found that

303 LN6 cells induced more migration of CXCR3+ Treg cells through the membrane

304 compared to parental cells (Figure 6g). With higher expression of CXCL10 and higher

305 frequency of lymph node metastasis in the LN6 line compared with parental line, this

306 finding is in accordance with our hypothesis that CXCR3-CXCL10 promotes Treg

307 migration toward malignant cells that colonize lymph nodes.

309 Given the existence of a small molecular weight antagonist, AMG487, that has shown to

310 block CXCR3 and reduce metastasis ${ }^{30-32}$, we hypothesized that Treg tumor infiltration is 
311 reduced following AMG487 treatment. We created an in vivo experiment to compare the

312 migration of Treg cells into parental tumor versus LN6 tumor with and without AMG487

313 treatment (Figure 6h). We found that LN6 tumors recruited more Tregs than the parental

314 line tumors in vivo (Figure 6i). Following AMG487 treatment on LN6 tumors, the

315 number of Tregs recruited into the tumor was reduced (Figure 6j), validating our

316 hypothesis.

\section{DISCUSSION}

319 Spatial biology is a new frontier that has become accessible through advances in 320 multiplexed in situ imaging. Exploring this frontier involves converting raw pixel-based

321 images into an interpretable cell-based format. This poses numerous technical challenges,

322 among them is the identification of cell types of individual cells. Common strategies for

323 cell type identification rely on manual gating or clustering algorithms. Gating and

324 clustering require substantial manual assessment, making them largely subjective, time

325 consuming and compromise single-cell resolution. We propose CELESTA, a novel

326 machine learning method tailored for identifying cell types on highly multiplexed

327 immunofluorescent in situ images by utilizing both the cell's protein expression profile

328 and spatial information. CELESTA is automated and fast $(\sim 10 \mathrm{~min}$ for $\sim 100 \mathrm{k}$ cells on a

329 typical Macbook).

331 CELESTA has several important features. CELESTA's input includes a cell-type

332 signature matrix of user-defined prior knowledge of the cell types, and a scoring function

333 that transfers this prior biological knowledge into cell type assignment in a non- 
335 specific marker is expressed or not in a cell, relative to the expression in all other cells;

336 this step relies on manual and subjective assessment in most existing cell type

337 identification methods. In the situation when the cell's protein expression profile is

338 insufficient to determine the cell type, CELESTA incorporates cell's spatial information

339 to identify the cell type, which has not been considered by existing methods. CELESTA

340 assigns the cell type to individual cells based on probabilities, and thus preserves the

341 single-cell resolution of the data. Embedded in CELESTA a "cell-type resolution"

342 strategy identifies cell types in multiple rounds based on cell lineage; this strategy not

343 only improves computational speed but also improves robustness when cell types from

344 different lineages have shared marker expression. CELESTA is able to handle large

345 dataset with fast assessment of the image. We have shown that CELESTA has

346 comparable performance against pathologist's manual assessment. For rare cell types that

347 are hard to identify, CELESTA's probabilities provide extra information for further

348 manual assessment. Lastly, we applied CELESTA to in situ images generated on a

349 multiplexed immunofluorescence-based platform, but CELESTA could also be extended

350 to data generated on other in situ imaging platforms.

352 Like other cell type identification methods, CELESTA requires segmenting cells in the

353 images as input and thus it relies on the performance of segmentation algorithm which

354 can be challenging with multiplexed imaging data. Particularly in the case of over-

355 segmentation, CELESTA may over-weight the spatial neighborhood information. Tissue

356 sample compositions and technical artifacts introduced by the imaging platform could 
add noise to the protein expression on some samples ${ }^{33,34}$. In such cases, some manual intervention is still needed to obtain more accurate cell type identification after CELESTA's fast assessment. CELESTA also relies on the cell-type marker information

360 in the user-defined input cell-type signature matrix. A poorly informed cell-type signature

361 matrix will effect the results. CELESTA, in its current version, does not account for cell

362 size or cell morphology, which are also important information for cell type identification.

364 We showed that CELESTA-informed geospatial tissue analysis revealed novel spatial

365 biology of HNSCC associated with lymph node metastasis, a finding which was validated

366 on an external cohort. We also reasoned that crosstalk between distinct cell types may be

367 associated with physical proximity ${ }^{24,25}$ and thereby identified spatial co-localization

368 patterns can provide guidance to discover potential cell-cell crosstalk mediators

369 associated with nodal status. Using spatially-guided scRNA-seq analysis, we identified

370 that the chemokine ligand-receptor pair CXCL10-CXCR3 have higher expressions on

371 malignant cells and Tregs respectively in N+ vs. N0 HNSCC. While CXCL10-CXCR3

372 crosstalk has been suggested to play a critical role in $\mathrm{T}$ cell trafficking and cancer

373 metastasis ${ }^{35-37}$, our work provides a potential role of CXCL10-CXCR3 axis in

374 mediating tissue architecture related to HNSCC lymph node metastasis. Using a small

375 molecule weight antagonist of CXCR3 that reduced Treg tumor infiltration, we

376 demonstrate the potential clinical importance of the CXCL10-CXCR3 axis as a

377 therapeutic target for metastatic HNSCC. In a similar manner we also identified the

378 CCR6-CCL20 axis, which was suggested to be related to cancer progression ${ }^{38-40}$,

379 mediating immune-endothelial crosstalk in node-positive disease. 
381 In summary, we propose CELESTA as an automated and fast cell type identification

382 method to facilitate multiplexed in situ image analysis, using both cells' protein

383 expression and spatial information. CELESTA enabled us to perform geospatial analysis

384 and identify important spatial biology associated with nodal status in HNSCC. Our work

385 also demonstrates the power of spatial biology to guide the discovery of cell-cell

386 interactions associated with disease progression and thereby provide new therapeutic

387 avenues.

$389 \quad$ METHODS

$390 \quad$ CELESTA

391 Scoring function: The first component in the algorithm is the scoring function to assess

392 how well a cell's marker expression profile match with our prior knowledge on the cell-

393 type markers. To apply the scoring function, we first need to quantify whether a marker is

394 expressed or not in a cell, which is a step often based on subjective assessment in most

395 existing cell type identification methods. We apply a two-mode Gaussian mixture model

396 to fit each marker's expressions across all the cells ${ }^{15}$ in a sample:

$$
f(x \mid \lambda)=\sum_{t}^{a} \phi_{t} g\left(x \mid \mu_{t}, \Sigma_{t}\right), a=\{0,1\}, \lambda=\{\phi, \mu, \Sigma\}
$$

398 Where $\phi$ is the mixing probabilities that sum up to one, $\mu$ is the mean and $\Sigma$ is the

399 covariance. If we assume that an expressed marker is in state $a=1$ and an unexpressed

400 marker is in state $a=0$, the posterior distributions for a marker to be expressed is $p(a=$

$401 \quad 1 \mid x)$ and unexpressed is $p(a=0 \mid x)$. At the decision boundary, we have:

$$
p(a=1 \mid x)=p(a=0 \mid x)
$$


By using Bayes' theorem, we obtain the following:

$$
p(x \mid a=1) p(a=1)=p(x \mid a=0) p(a=0)
$$

$405 \quad$ where $p(x \mid a=1)=g\left(x \mid \mu_{1}, \Sigma_{1}\right)$ and $p(x \mid a=0)=g\left(x \mid \mu_{0}, \Sigma_{0}\right) \cdot p(a=1)$ and

$406 p(a=0)$ are the mixing probability $\phi_{1}$ and $\phi_{0}$. All could be obtained from the Gaussian

407 mixture model. By solving equation (3), we identify the decision critical point $x_{c}$ at

408 which a marker has equal probabilities for expressing versus not expressing. We thus

409 could use a logistic function to quantify a marker expression probability $(E P)$ for each

410 marker in each cell as:

$$
E P(x)=\frac{1}{1+\exp \left(-\left(x-x_{c}\right)\right)}
$$

412 We repeat the process for every marker, and thus for each cell, its protein marker

413 expressions are converted into expression probabilities. With the reference expression

414 probabilities for markers expressed or not expressed for a cell type defined in the cell-

415 type signature matrix for respective cell types, we define our scoring function for a cell

416 type as one minus the mean squared error between each cell's marker expression

417 probabilities and the reference expressions in the cell-type signature matrix for that cell

418 type. When a cell has one dominate cell type score, CELESTA assigns the corresponding

419 cell type to that cell and defines the cell as an "anchor cell". For a cell whose cell type

420 cannot be identified using protein expression alone, it is defined as an index cell. In

421 addition, a threshold for expressing and a threshold for not expressing based on the

422 expression probability are also required. For example, we can set the high threshold to be

$423 \quad 0.7$ and low threshold to be 0.3 for tumor cells. This means that for a cell to be tumor cell,

424 it needs to have cytokeratin (tumor marker) expression probability higher than 0.7 and all

425 other non-expressing protein marker expression probability lower than 0.3 , in addition to 
426 having a scoring function producing high score on tumor cell compare to other cell types.

427 The thresholds provide user the flexibility to define artifacts like doublets or staining

428 background noise. The cell-type signature matrix is updated in each iteration to

429 incorporate the averaging expression probabilities from cells that are assigned cell types

$430 \quad$ into the reference expressions.

432 CELESTA employs an optimization framework to assign the most likely cell type to each

433 index cell. CELESTA is designed to maximize the joint probability distribution using

434 hidden Markov Random Field (MRF) ${ }^{41}$ that includes an energy function component that

435 accounts for cell spatial information and a scoring function component that accounts for

436 protein marker expressions. We assume each cell $C$ whose cell type needs to be inferred

437 (index cell) is a node in an undirected graph and each cell has connected neighboring

438 cells that are stochastically dependent. We model the stochastic spatial dependency using

439 neighboring system defined on the undirected graph $G$ with the edges connecting the cell

440 to its N-nearest neighboring cells. Depending on the cell densities in the tissue, we

441 recommend $\mathrm{N}=5 \sim 10$. We associate each node (an index cell) with an unknown state $S$

442 which is the cell type to be inferred. Thus, the spatial dependency on the undirected graph

443 is modeled by hidden Markov Random Field (MRF) with joint probability distribution of

444 Gibbs distribution as:

445

$$
P_{G}(\boldsymbol{S} ; \boldsymbol{\beta})=W(\boldsymbol{\beta})^{-1} \exp (-E(\boldsymbol{S} ; \boldsymbol{\beta}))
$$

446 Where $\boldsymbol{\beta}$ is a set of model parameters to be estimated, $W(\boldsymbol{\beta})$ is a normalization constant, 447 and $E$ is the energy function. 
Energy function: For our energy function $E$, we use the Potts model ${ }^{17,18}$, defined as:

$$
E\left(s_{i} ; \boldsymbol{\beta}\right)=-\boldsymbol{\beta} \sum_{i \sim j}^{N} 1\left[s_{i}=s_{j}\right]
$$

451 Where $N$ is the number of nearest spatial neighboring cells of the index cell $i$ based on

452 cells' $\mathrm{X}$ and Y coordinates obtained from the image. It is an indicator function. If an

453 index cell has the same cell type as a neighbor cell $j$, one is added to the summation and

454 thus increase the probability for that cell type. $\boldsymbol{\beta}$ is a set of model parameters depending

455 on distances between cells. $\boldsymbol{\beta}$ is used to decide how much information to include from

456 the neighboring cells, defined by triangular kernel times a user defined scale factor $\gamma$ as:

$$
\beta_{i k}=\gamma \times\left(1-\frac{d_{i k}}{h}\right) \text { for } \frac{d_{i k}}{h}<1 \text {. Otherwise, } \beta_{i k}=0
$$

458 where $d_{i k}$ is the distance between index cell $i$ and its nearest cell that has cell type $k$

459 assigned, and $h$ is the bandwidth. Therefore, if there are no cells of cell type $k$ assigned

460 within distance $h$ to the index cell, no neighborhood information from cell type $k$ is used.

461 If an index cell is too far away from any cells with cell types identified, no spatial

462 neighborhood information is used for that cell, which could help accounting for isolated

463 cells. CELESTA uses the spatial dependency term to account for the situation where an

464 index cell's protein expression profile has similar scores matched to multiple cell types,

465 reasoning that cells with the same cell type are enriched within each other's spatial

466 neighborhoods.

467

468 Optimization of objective function for cell type identification: With both the energy

469 function and scoring function defined, our objective function is expressed as the overall

470 joint probability distribution:

471

$$
P(\boldsymbol{S} ; \boldsymbol{\beta})=W(\boldsymbol{\beta})^{-1} \exp \left(-E(\boldsymbol{S} ; \boldsymbol{\beta})+\sum_{i \in n} \boldsymbol{F}\right)
$$


472 where $n$ is the total number of index cells. $E$ is the energy function component and $F$ is

473 the scoring function component. We intend to assign each individual cell with the cell

474 types that maximize the joint probability distribution. Because our objective function is

475 non-convex, we use a pseudo-EM algorithm to iteratively solve the problem. In the

476 initialization step, we only use the scoring function to identify cells whose protein marker

477 expressions could be well matched to only one cell type based on prior knowledge in

478 cell-type signature matrix, and define those cells as anchor cells and assign the matched

479 cell types to the anchor cells. Each iteration, we approximate the probability of cell type

$480 \quad k$ for an index cell $i$ using mean field approximation ${ }^{19}$ by:

$$
P_{i k}=\frac{S_{i k} \times E\left(y_{i k} \mid y_{N(i)}, \beta\right)}{\sum_{u}^{K} S_{i u} \times E\left(y_{i u} \mid y_{N(i)}, \beta\right)}
$$

482 where $K$ is total number of cell types in the cell-type signature matrix. If there are

483 multiple rounds defined in the prior knowledge using "cell-type resolution" strategy, $K$ is

484 the total number of cell types in a round. $N$ is the total number of neighbors of index cell

$485 \quad i$. Essentially, we approximate probabilities of each cell type for the index cell and assign

486 the cell type with dominating probability to the index cell. A threshold of the probability

487 is required. If the cell type probabilities do not pass the threshold, then no cell type is

488 assigned for that cell in this current iteration and the cell is carried over to next iteration

489 with more cell types assigned and increased neighborhood information. Each iteration,

490 we update the parameters of $\beta$, the cell-type signature matrix, the scoring function and

491 the energy function based on the new neighborhood information. The convergence

492 condition is defined as the percentage of cells that do not have cell type assignment is

493 smaller than a user-defined threshold. After the convergence condition is met, if a cell is

494 still not assigned to a cell type, we assign the cell with an "Unknown" cell type. 


\section{Human tumor specimens}

497 All patients at the Stanford Hospital included were consented to take part in the study

498 following Institutional Review Board (IRB) approval (IRB protocol \#: 11402). The

499 staging information of the patient samples are summarized in Table S2. Fresh tissue of

500 head and neck squamous cell carcinoma (HNSCC) was collected within six hours of

501 surgical resection. A two-to-three millimeter tissue piece was cut from the sample.

502 Patient 7153, and 7155 OCT samples were immediately frozen in OCT freezing media,

503 while patient $7233,7238,7240,7267,7268$ and 7275 OCT samples were placed in $30 \%$

504 sucrose for 1 hour at $4^{\circ}$ Celsius and frozen in OCT freezing media (Fisher Healthcare,

505 Houston, TX) on a metal block chilled in liquid nitrogen. OCT samples were stored in -

$50680^{\circ} \mathrm{C}$ for CODEX processing and sequencing. The remaining tissue was placed on ice

507 and in $50 \mu$ lissue digestion media, DMEM-F12+ with magnesium and calcium (Corning

508 Cellgro, Manassas, VA), 1\%FBS (heat inactivated), 10units/ml Penicillin-10ug/ml

509 Streptomycin (Gibco, Grand Island, NY), 25mM hepes (Gibco, Grand Island, NY).

510

511 CODEX image acquisition and segmentation

512 Multiplexed CODEX analysis of HNSCC tissues was performed using a panel of

513 antibodies (Table S3) conjugated to custom DNA barcodes and detector oligos as well as

514 common buffers, robotic imaging setup and instructions for CODEX staining of frozen

515 specimen from Akoya Biosciences (https://www.akoyabio.com/). 7um sections were cut

516 with a cryostat after OCT blocks were equilibrated to the cryostat temperature for at least

$517 \quad 30-40$ minutes. Tissue sections were placed on the surface of cold poly-L-lysine coated 
518 coverslips and adhered by touching a finger to the bottom surface to transiently warm up

519 the coverslip. Frozen sections on coverslips can be stored at $-70 \mathrm{C}$ for $1-2$ months.

520 Section pre-processing was done as previously described ${ }^{14}$. Prior to staining the sections,

521 frozen sections removed from the freezer were dried for $5 \mathrm{~min}$ on the surface of Drierite.

522 Dried coverslips with sections on them were dipped for $10 \mathrm{~min}$ into room temperature

523 acetone, then fully dried for $10 \mathrm{~min}$ at room temperature. Sections were then rehydrated

524 for $5 \mathrm{~min}$ in S1 [5 mM EDTA (Sigma), 0.5\% w/v bovine serum albumin (BSA, Sigma)]

525 and $0.02 \% \mathrm{w} / \mathrm{v}$ NaN3 (Sigma) in PBS (Thermo Fisher Scientific) then fixed for 20 min at

526 room temperature in S1 with $1.6 \%$ formaldehyde. Formaldehyde was rinsed off twice

527 with S1. Sections were equilibrated in S2 [61 mM NaH2PO4 · 7 H2O (Sigma)], $39 \mathrm{mM}$

$528 \mathrm{NaH} 2 \mathrm{PO} 4$ (Sigma) and $250 \mathrm{mM} \mathrm{NaCl}$ (Sigma) in a 1:0.7 v/v solution of S1 and doubly-

529 distilled $\mathrm{H} 2 \mathrm{O}(\mathrm{ddH} 2 \mathrm{O})$; with final $\mathrm{pH}$ of 6.8-7.0 for 10 minutes, and placed in blocking

530 buffer for 30 minutes. All steps to follow were exactly as previously described ${ }^{14}$ or per

531 the Akoya CODEX instructions.

533 Automated image acquisition and fluidics exchange were performed using an Akoya

534 CODEX instrument driven by CODEX driver software (Akoya Biosciences) and

535 Keyence BZ-X710 fluorescent microscope configured with 4 fluorescent channels

536 (DAPI, FITC, Cy3, Cy5) and equipped with CFI Plan Apo $\lambda$ 20x/0.75 objective (Nikon).

537 Hoechst nuclear stain (1:3000 final concentration) was imaged in each cycle at an

538 exposure time of 1/175s. Biotinylated CD39 - detection reagent was used at a dilution of

$5391: 500$, and visualized in the last imaging cycle using DNA streptavidin- PE (1:2500 final

540 concentration). DRAQ5 nuclear stain (1:500 final concentration) was added and 
541 visualized in the last imaging cycle. Each tissue was imaged with a 20x objective in a $7 x 9$

542 tiled acquisition at 1386x1008 pixels per tile and $396 \mathrm{~nm} /$ pixel resolution and $13 \mathrm{z}$-planes

543 per tile (axial resolution $1500 \mathrm{~nm}$ ). Images were subjected to deconvolution to remove

544 out-of-focus light. Acquired images were pre-processed (alignment and deconvolution

545 with Microvolution software (http://www.microvolution.com/)) and segmented

546 (including lateral bleed compensation) using publicly available CODEX image

547 processing pipeline available at https://github.com/nolanlab/CODEX.

549 Manual assessment of CELESTA performance on the HNSCC cohort

550 CELESTA performance on each sample of the HNSCC cohort was assessed manually by

551 mapping CELESTA assigned cell types onto the CODEX images using the X and Y

552 coordinates (Figure S2). For each cell type, CELESTA assigned cells were plotted as

553 yellow crosses on the canonical marker staining images. Marker staining was shown as

554 white signals on black background. Assessment for each cell were defined as positive

555 canonical marker signals for that cell type. Manual assessment on CELESTA cell type

556 assignments showed over $90 \%$ positive signals across the cell types on different samples.

$558 \quad$ Manual gating of HNSCC cohort

559 The segmented CODEX HNSCC dataset was uploaded onto the Cytobank analysis

560 platform and transformed with an inverse hyperbolic sine (asinh) transformation (cofactor

561 of 5). The gating strategy used was as follows (Figure S3): Cells were defined by

562 DRAQ5 nuclear expression and size, followed by endothelial cells (CD31+) and

563 malignant cells (Cytokeratin+). CD4+ T cells (CD4+ CD8- CD3+ CD31- Cytokeratin-), 
564 CD8+ T cells (CD8+ CD4- CD3+ CD31- Cytokeratin-), CD4/CD8 double positive cells

565 (CD8+CD4+CD3+CD31-Cytokeratin-), CD4/CD8 double negative cells (CD8-CD4-

566 CD3 + CD31- Cytokeratin-), and T regulatory cells (FOXP3+ CD25+ CD4+ CD8- CD3+

567 CD31- Cytokeratin-) were then defined. To adjust for the variability between sample

568 image collection, each gate was tailored to each individual sample.

569

$570 \quad$ Spatial analysis

571 We used co-location quotient (CLQ) ${ }^{23}$ to identify cell spatial co-localization. If we

572 define cell type $a$ as target cells and cell type $b$ as neighboring cells, the CLQ is the

573 degree of cell type $b$ co-locates with cell type $a$ as ratio of the observed versus expected

574 number of cell-type $b$ among the set of nearest neighbors of cell-type $a$ defined as:

$575 \quad C L Q_{b \rightarrow a}=\frac{C_{b \rightarrow a} / N_{a}}{N_{b} /(N-1)}$

576 Where $C$ is the number of cells of cell type $b$ among the defined nearest neighbors of cell

577 type $a . N$ is the total number of cells and $N_{a}$ and $N_{b}$ are the numbers of cells for cell type

$578 \quad a$ and cell type $b$. Cell types with fewer than twenty cells were excluded for each sample.

579 We calculated a CLQ for every pair-wise cell types identified for each sample, and

580 compared the CLQs on each pair of cell types between N+ and N0 samples to identify

$581 \quad$ significantly different CLQs.

582

$583 \quad$ Tumor tissue dissociation

584 Tumor tissue was thoroughly minced with a sterile scalpel and placed in a gentleMACS

585 C-tube (Miltenyi Biotec, Sunnyvale, CA) containing 1.5mls of tissue digestion media.

586 Tissue was mechanically digested on the GentleMACS dissociator five times under the 
human tumor tissue program h_tumor_01. Tissue was filtered with a $40 \mu \mathrm{m}$ nylon cell

588 strainer (Falcon, Corning, NY) into a $14 \mathrm{ml}$ tube filled up to $14 \mathrm{mls}$ of tissue digestion

589 media and spun at $4^{\circ} \mathrm{C}$ for $10 \mathrm{~min}$ at $514 \mathrm{RCF}$. The mechanically digested cell pellet was

590 re-suspended for 2 minutes on ice in 1-4 mls of ACK lysis buffer (Gibco, Grand Island,

591 NY) depending on the pellet size and number of red blood cells present. Cells were

592 filtered with a $40 \mu \mathrm{m}$ nylon cell strainer (Falcon, Corning, NY) into a $14 \mathrm{ml}$ tube filled up

593 to $14 \mathrm{mls}$ of FACS buffer, Phosphate Buffered Saline without calcium or magnesium

594 (Corning, Manassas, VA), 2\%FBS heat inactivated, 10units/ml Penicillin-10ug/ml

595 Streptomycin (Gibco, Grand Island, NY), 1mM Ultra Pure EDTA (Invitrogen, Carlsbad,

$596 \mathrm{CA}$ ) and spun at $4^{\circ} \mathrm{C}$ for $10 \mathrm{~min}$ at 514RCF. Cells were washed one more time with

$597 \quad$ FACS buffer and re-suspended in $25 \mu$ l of FACS buffer. Solid tissue in the strainer was

598 collected and placed back in the C-tube with $2 \mathrm{mls}$ of tissue digestion media, $1 \mathrm{ml}$ of

$5993000 \mathrm{U} / \mathrm{ml}$ collagenase/1000U/ml hyaluronidase (StemCell Technologies, Vancouver,

$600 \mathrm{BC}$ ) and $1 \mathrm{ml}$ of 5U/ml dispase (StemCell Technologies, Vancouver, BC). The solid

601 tissue in the C-tube was incubated at $37^{\circ}$ Celsius on a rotator for 1 hour, then filtered

602 with a $40 \mu \mathrm{m}$ nylon cell strainer (Falcon, Corning, NY) into a $14 \mathrm{ml}$ tube filled up to 14

$603 \mathrm{mls}$ of tissue digestion media and spun at $4^{\circ} \mathrm{C}$ for $10 \mathrm{~min}$ at $514 \mathrm{RCF}$. The enzymatically

604 digested cell pellet was re-suspended in 1-4 mls of ACK lysis buffer (Gibco, Grand

605 Island, NY) depending on the pellet size and number of red blood cells present for 2

606 minutes on ice. Cells were filtered with a $40 \mu \mathrm{m}$ nylon cell strainer (Falcon, Corning,

607 NY) into a $14 \mathrm{ml}$ tube filled up to $14 \mathrm{mls}$ of FACS buffer, (Phosphate Buffered Saline

608 without calcium or magnesium (Corning, Manassas, VA), 2\%FBS heat inactivated,

609 10units/ml Penicillin-10ug/ml Streptomycin (Gibco, Grand Island, NY), 1mM Ultra pure 
$610 \quad$ EDTA (Invitrogen, Carlsbad, CA) and spun at $4^{\circ} \mathrm{C}$ for $10 \mathrm{~min}$ at 514RCF. Cells were re-

611 suspended in FACS buffer, counted on a hemacytometer and washed one more time with

612 FACS buffer. Cells were kept in FACS buffer on ice until flow cytometry staining.

613 Sorting panel is shown in supplement Table S5.

614

$615 \quad$ Single-cell RNA sequencing

616 RNA and library preparations were performed for 10x Genomics scRNA-sequencing

617 samples according to $10 \mathrm{x}$ Genomics vs 2.0 handbook. Single cells were obtained

618 according to the previous tumor tissue dissociation. Cells were stained with DAPI for

619 live/dead detection and sorted for up to 500,000 live cells on a BD Aria II. Cells were

620 counted after sort and right before 10x chip prep. 10x/Abseq by BD biosciences,

621 followed the same protocol as the 10x Genomics samples except for the addition of

622 FcBlock and Abseq antibody staining according to the manufacturer's handbook.

623

$624 \quad$ Singe-cell RNA-sequencing data processing and analysis

625 We aligned the reads using CellRanger. Preprocessing, data normalization and batch

626 correction were done following Seurat SCTransform integration pipeline. UMAPs were

627 obtained by using top 50 principal components. Cells were clustered by shared nearest

628 neighbor modularity optimization. Cell types present were identified with canonical

629 markers. Differentially expressed genes were identified in each cell type cluster between

$630 \mathrm{~N} 0$ and $\mathrm{N}+$ patients using permutation test, and false discovery rate was used for multiple

631 testing correction.

632 


\section{Tissue microarray of HNSCC}

634 Formalin-fixed paraffin-embedded tissue blocks of head and neck squamous cell

635 carcinoma from 79 patients were pulled from the Stanford Health Care Department of

636 Pathology archives. The area of malignancy was marked by a board-certified (C.S.K.)

637 pathologist. Tissue microarrays were constructed from $0.6 \mathrm{~mm}$ diameter cores punched

638 from the tissue blocks. 4 um thick sections were stained with hematoxylin and eosin,

639 FOXP3 (clone 236A/E7, 1:100 dilution; Leica BOND epitope retrieval solution 2) and

640 cytokeratin mix (AE1/AE3, 1:75 dilution \& CAM5.2, 1:25 dilution; Ventana Ultra;

641 protease retrieval). The slides were digitized using Leica whole slide scanner with 40x

642 magnification. Three samples with unknown lymph node status were excluded from

$643 \quad$ analysis.

644

645 To assess co-localization of FOXP3 and cytokeratin immunohistochemistry staining, a

646 preprocessing pipeline was built for the TMA data. The whole-slide images were

647 dearrayed to obtain each core image. We next ran color deconvolution to quantify DAB

648 staining using "scikit-image" package in Python. We thresholded the staining based on

649 pixel intensity distributions of the DAB staining to quantify positive stained pixels in the

650 images. We used a sliding window with 100 by 100 pixels to quantify the positive pixel

651 densities for cytokeratin and FOXP3 within each window and move the sliding windows

652 to cover the whole core area. We correlated the densities of cytokeratin staining and

653 FOXP3 staining across sliding windows for each sample. We compared the density

654 correlations between N0 and N+ samples.

655 


\section{$656 \quad$ Statistical analysis and figure creation}

657 Statistical analyses were performed and corresponding figures were generated in R or

658 Python depending on the analysis packages. The student's t-test, Wilcoxon rank-sum test

659 and permutation test were utilized for comparisons between $\mathrm{N} 0$ and $\mathrm{N}+$ samples.

660 Specifically, for human patient data, student's t-test was used for co-location quotient

661 comparisons and density correlation comparisons between N0 and N+ sample. For in

662 vitro and in vivo functional experiments, non-parametric Wilcoxon rank-sum test was

663 used for comparisons. In addition, we created a paired-group setup in the transwell assay

664 experiment and used paired test for statistical analysis. For multiple testing, permutation

665 test was used and false discovery rate was used to adjust the p-values. Results were

666 considered statistically significant when $\mathrm{p}<0.05$ or adjusted $\mathrm{p}<0.05$ for multiple testing.

667 Parts of Figure 5 and 6 were created using Biorender online tool (https://biorender.com).

668 Multi-channel overlay images were created using ImageJ.

669

670 For the public colorectal cancer dataset, the "ground truth" is defined using the

671 annotations in the publication ${ }^{6}$. For the study cohort of HNSCC dataset, the "ground

672 truth" standard is defined using manual gating based on gating strategies on cell types

673 relevant to downstream spatial analysis. For each cell type, true positives (TP) is the

674 number of cells assigned by both CELESTA and ground truth benchmark. False positives

675 (FP) is the number of cells which were assigned by CELESTA but not ground truth

676 benchmark. False negatives (FN) is the number of cells assigned in benchmark but not

677 CELESTA. True negatives (TN) is the number of cells that are not assigned as the cell

678 type by both CELESTA and benchmark. Precision is defined as TP/(TP+FP), and recall 
679 is defined as $\mathrm{TP} /(\mathrm{TP}+\mathrm{FN})$. F1 score is defined as $2($ precision*recall)/(precision+recall).

680 Rand index to measure accuracy is defined as $(\mathrm{TP}+\mathrm{TN}) /(\mathrm{TP}+\mathrm{TN}+\mathrm{FP}+\mathrm{FN})$. For the

681 public colorectal cancer dataset, cell types with fewer than 5 cells in a sample region in

682 the annotations were excluded. For HNSCC study cohort, adjusted rand index (ARI) was

683 calculated for each sample using R package "mclust" adjustedRandIndex function.

684

$685 \quad$ Cell lines and animals

686 B16-F0 parental and LN6-987AL murine melanoma cell lines have been described

687 previously ${ }^{29}$. Cells were cultured in Dulbecco's Modified Eagle Medium (DMEM)

688 supplemented with 4mM L-glutamine, 10\% Fetal Bovine Serum (FBS), and 1\%

689 Penicillin Streptomycin. The tumor lines were routinely tested for mycoplasma by PCR,

690 and all tests were negative. All animal studies were performed in accordance with the

691 Stanford University Institutional Animal Care and Use Committee under protocol

692 APLAC-17466. All mice were housed in an American Association for the Accreditation

693 of Laboratory Animal Care-accredited animal facility and maintained in specific

694 pathogen-free conditions.

695

$696 \quad$ Transwell migration assays

697 FoxP3 ${ }^{\text {EGFP }}$ mice ${ }^{42}$ were acquired from Jackson (006772) and bred in our facility at

698 Stanford University. Splenocytes were harvested from tumor-naive female FoxP3 ${ }^{\text {EGFP }}$

699 mice. Spleens were subjected to mechanical dissociation on $70 \mu \mathrm{m}$ cell strainers and

700 washed with HBSS supplemented with 2\% FBS and 2mM Ethylenediaminetetraacetic

701 acid (EDTA) (HBSSFE). Erythrocytes were lysed with Ammonium-Chloride-Potassium 
(ACK). Magnetic isolation of Tregs was performed using the EasySep Mouse CD25

703 Regulatory T cell Positive Selection Kit (StemCell, 18782) according to the

704 manufacturer's instructions. Tregs were cultured in RPMI-1640 supplemented with 10\%

705 FBS, 2mM L-glutamine, 15mM HEPES, $14.3 \mathrm{mM}$ 2-mercaptoethanol, 1mM Sodium

706 Pyruvate, $1 \times$ MEM Non-Essential Amino Acids Solution, and 300IU hIL-2 (Peprotech)

707 for 72 hours.

708

709 Tumor cell line suspensions were prepared by washing with phosphate buffered saline

710 (PBS) followed by treatment with StemPro Accutase (Thermo, A1110501). 10 $0^{5}$ tumor

711 cells were plated in the bottom chamber of the 24 -well transwell plates 24 hours prior to

712 the assay. $5 \mu \mathrm{m}$ transwell membranes (Costar, 3421) were incubated in complete RPMI

713 for 24 hours prior to the assay. Membranes were transferred to the tumor-containing

714 wells and suspensions of $5 \times 10^{4}$ Tregs were added to the top chambers of the transwells.

715 Cells were cultured for 2 hours at $37^{\circ} \mathrm{C}$ in $5 \% \mathrm{CO}_{2}$, after which the membranes were

716 removed, and cells from the bottom chamber were processed for analysis by flow

717 cytometry.

718

719 Cell suspensions were washed in HBSSFE and stained with the following antibodies:

720 Mouse Fc Block (BD, 2.4G2), CD4 (BioLegend, RM4-5), CD25 (BioLegend, PC61), and

721 CXCR3 (BioLegend, CXCR3-173). 4',6-Diamidino-2-Phenylindole Dihydrochloride

722 (DAPI) was used to stain for viability. Samples were run on an LSRFortessa cytometer

723 (Becton Dickinson) and analyzed using FlowJo V10 software (TreeStar). 


\section{In vivo Treg tumor infiltration}

726 Experiments were performed using either C57NL/6J (Jackson, 000664) or FoxP3 ${ }^{\text {EGFP }}$

727 (Jackson, 006772) female mice housed in our facility at Stanford. B16-F0 or LN6-987AL

728 tumor cells were washed with PBS and dissociated from tissue culture plastic with

729 StemPro Accutase (Thermo, A1110501). Cell suspensions of $2 \times 10^{5}$ cells in phenol-red

730 free DMEM were injected into the subcutaneous region of the left flank of nine-week-old

731 female mice (Jackson, 000664) following removal of fur with surgical clippers. After 15

732 days of tumor growth, mice were euthanized and their tumors were processed for analysis

733 by flow cytometry.

734

735 Tumors were weighed followed by digestion in RPMI-1640 supplemented with $4 \mathrm{mg} / \mathrm{mL}$

736 Collagenase Type 4 (Worthington, LS004188) and 0.1mg/mL Deoxyribonuclease I

737 (DNAse I, Sigma, DN25) at $37^{\circ} \mathrm{C}$ for 20 minutes with agitation. Tumors were then

738 dissociated on $70 \mu \mathrm{m}$ strainers, washed with HBSSFE, and stained for viability using

739 LIVE/DEAD Fixable Blue Dead Cell Stain (Thermo, L34962). Surface proteins were

740 stained, samples were fixed and permeabilized using the eBioscience FoxP3

741 Fixation/Permeabilization kit (Thermo, 00-5521-00), and intracellular FoxP3 was stained.

742 The following antibodies were used: Mouse Fc Block (BD, 2.4G2), CD4 (BioLegend,

743 RM4-5), CD8 $\alpha$ (BioLegend, 53-6.7), CD3 (BioLegend, 17A2), CD25 (BioLegend,

744 PC61), CXCR3 (BioLegend, CXCR3-173), B220 (BioLegend, RA3-6B2), CD45.2

745 (BioLegend, 104), and FoxP3 (Thermo/eBiosciences, NRRF-30). AccuCount Fluorescent

746 particles (Spherotec, ACFP-50-5) were added to each samples for the purposes of 
747 determining absolute cell counts. Samples were run on an LSRFortessa cytometer

748 (Becton Dickinson) and analyzed using FlowJo V10 software (TreeStar).

750 For treatment CXCR3-blockade studies, LN6-987AL cells were prepared as above and

751 injected into seven-week-old FoxP3 ${ }^{\text {EGFP }}$ mice. Mice were treated with AMG487 (R\&D

752 Systems, 4487) at $5 \mathrm{mg} / \mathrm{kg}$ every $48 \mathrm{hrs}$ starting on day one following tumor implantation.

753 After 9 days of tumor growth, mice were euthanized and their tumors were processed for

$754 \quad$ analysis by flow cytometry as described above.

755

756 ACKNOWLEDGEMENT

757 This work was supported by the National Institute of Health, National Cancer Institute

$758 \quad$ U54 CA209971.

759

760 DATA AND CODE AVAILABILITY

761 The scRNA-seq data are deposited at GEO: GSE140042. HNSCC imaging data are

762 hosted at Synapse.org SageBionetworks at https://doi.org/10.7303/syn26242593. The

763 benchmark public imaging data can be found at https://doi.org/10.7937/tcia.2020.fqn0-

764 0326. All codes related to CELESTA can be found at

765 https://github.com/plevritis/CELESTA.

767 REFERENCES

$768 \quad$ 1. Stack, E. C., Wang, C., Roman, K. A. \& Hoyt, C. C. Multiplexed

769 immunohistochemistry, imaging, and quantitation: A review, with an assessment 
of Tyramide signal amplification, multispectral imaging and multiplex analysis.

772 2. Angelo, M. et al. Multiplexed ion beam imaging (MIBI) of human breast tumors.

$773 \quad$ Nat. Med. 20, 436-442 (2014).

774 3. Wang, Y. J. et al. Multiplexed in situ imaging mass cytometry analysis of the

775 human endocrine pancreas and immune system in type 1 diabetes. Cell Metab. 29, 776 769-783.e4 (2019).

777 4. Ptacek, J. et al. Multiplexed ion beam imaging (MIBI) for characterization of the

778 tumor microenvironment across tumor types. Lab. Investig. 100, 1111-1123

779 (2020).

780 5. Parra, E. R., Francisco-Cruz, A. \& Wistuba, I. I. State-of-the-art of profiling 781 immune contexture in the era of multiplexed staining and digital analysis to study 782 paraffin tumor tissues. Cancers (Basel). 11, (2019).

783 6. Schürch, C. M. et al. Coordinated cellular neighborhoods orchestrate antitumoral 784 immunity at the colorectal cancer invasive front. Cell 182, 1341-1359.e19 (2020).

785 7. Gillies, R. J., Verduzco, D. \& Gatenby, R. A. Evolutionary dynamics of 786 carcinogenesis and why targeted therapy does not work. Nat. Rev. Cancer 12, 487$487(2012)$

788 8. Heindl, A., Nawaz, S. \& Yuan, Y. Mapping spatial heterogeneity in the tumor 789 microenvironment: A new era for digital pathology. Lab. Investig. 95, 377-384 790 (2015).

791 9. Alfarouk, K. O., Ibrahim, M. E., Gatenby, R. A. \& Brown, J. S. Riparian 792 ecosystems in human cancers. Evol. Appl. 6, 46-53 (2013). 
10. Little, S. E. et al. Receptor tyrosine kinase genes amplified in glioblastoma exhibit a mutual exclusivity in variable proportions reflective of individual tumor heterogeneity. Cancer Res. 72, 1614-1620 (2012).

796 11. Herzenberg, L. A., Tung, J., Moore, W. A., Herzenberg, L. A. \& Parks, D. R. Interpreting flow cytometry data: A guide for the perplexed. Nat. Immunol. 7, 681685 (2006).

12. Aghaeepour, N. et al. Critical assessment of automated flow cytometry data analysis techniques. Nat. Methods 10, 228-238 (2013).

801 13. Shekhar, K., Brodin, P., Davis, M. M. \& Chakraborty, A. K. Automatic classification of cellular expression by nonlinear stochastic embedding (ACCENSE). Proc. Natl. Acad. Sci. U. S. A. 111, 202-207 (2014).

804 14. Goltsev, Y. et al. Deep profiling of mouse splenic architecture with CODEX 805 multiplexed imaging. Cell 174, 968-981.e15 (2018).

806 15. Lee, H. C., Kosoy, R., Becker, C. E., Dudley, J. T. \& Kidd, B. A. Automated cell 807 type discovery and classification through knowledge transfer. Bioinformatics 33, 808 1689-1695 (2017).

809 16. Pettit, J. B. et al. Identifying cell types from spatially referenced single-cell

$810 \quad$ expression datasets. PLoS Comput. Biol. 10, e1003824 (2014).

811 17. Wu, F. Y. The Potts Model. Rev. Mod. Phys. 54, (1982).

812 18. Storath, M., Weinmann, A., Frikel, J. \& Unser, M. Joint image reconstruction and 813 segmentation using the Potts model. Inverse Probl. 31, (2015).

814 19. Celeux, G., Forbes, F. \& Peyrard, N. EM-based image segmentation using Potts 815 models with external field. INRIA (2002). 
816 20. Li, Q., Yi, F., Wang, T., Xiao, G. \& Liang, F. Lung cancer pathological image

817 analysis using a hidden potts model. Cancer Inform. 16, (2017).

818 21. Celeux, G. et al. EM procedures using mean field-like approximations for Markov

819 model-based image segmentation. Pattern Recognit. 36, 131-144 (2003).

820 22. Samusik, N., Good, Z., Spitzer, M. H., Davis, K. L. \& Nolan, G. P. Automated

821 mapping of phenotype space with single-cell data. Nat. Methods 13, 493-496

822 (2016).

823 23. Leslie, T. F. \& Kronenfeld, B. J. The colocation quotient: A new measure of

824 spatial association between categorical subsets of points. Geogr. Anal. 43, 306-

$825 \quad 326(2011)$.

826 24. Arnol, D., Schapiro, D., Bodenmiller, B., Saez-Rodriguez, J. \& Stegle, O.

827 Modeling cell-cell interactions from spatial molecular data with spatial variance

828 component analysis. Cell Rep. 29, 202-211.e6 (2019).

829 25. Ramilowski, J. A. et al. A draft network of ligand-receptor-mediated multicellular

$830 \quad$ signalling in human. Nat. Commun. 6, 1-11 (2015).

831 26. Butler, A., Hoffman, P., Smibert, P., Papalexi, E. \& Satija, R. Integrating single-

832 cell transcriptomic data across different conditions, technologies, and species. Nat.

$833 \quad$ Biotechnol. 36, 411-420 (2018).

834 27. Stuart, T. et al. Comprehensive Integration of Single-Cell Data. Cell 177, 1888-

835 1902.e21 (2019).

836 28. Puram, S. V. et al. Single-cell transcriptomic analysis of primary and metastatic

837 tumor ecosystems in head and neck cancer. Cell 171, 1611-1624.e24 (2017).

838 29. Reticker-Flynn, N. et al. Lymph node colonization alters the systemic immune 
response to enable metastasis to distant tissues. Cell Sneak Peek 2.0 (2020).

840 30. Zhu, G. et al. CXCR3 as a molecular target in breast cancer metastasis: Inhibition

841 of tumor cell migration and promotion of host anti-tumor immunity. Oncotarget 6,

$842 \quad 43408-43419(2015)$.

843 31. Cambien, B. et al. Organ-specific inhibition of metastatic colon carcinoma by

844 CXCR3 antagonism. Br. J. Cancer 100, 1755-1764 (2009).

845 32. Walser, T. C. et al. Antagonism of CXCR3 inhibits lung metastasis in a murine 846 model of metastatic breast cancer. Cancer Res. 66, 7701-7707 (2006).

847 33. Kim, D., Curthoys, N. M., Parent, M. T. \& Hess, S. T. Bleed-through correction 848 for rendering and correlation analysis in multi-colour localization microscopy. $J$.

$849 \quad$ Opt. 15, 1-30 (2013).

850 34. Rich, R. M. et al. Elimination of autofluorescence background from fluorescence 851 tissue images by use of time-gated detection and the AzaDiOxaTriAngulenium 852 (ADOTA) fluorophore. Anal. Bioanal. Chem. 405, 2065-2075 (2013).

853 35. Groom, J. R. \& Luster, A. D. CXCR3 in T cell function. Exp. Cell Res. 317, 620$854631(2011)$.

855 36. Wightman, S. C. et al. Oncogenic CXCL10 signalling drives metastasis

856 development and poor clinical outcome. Br. J. Cancer 113, 327-335 (2015).

857 37. Tokunaga, R. et al. CXCL9, CXCL10, CXCL11/CXCR3 axis for immune 858 activation - A target for novel cancer therapy. Cancer Treat. Rev. 63, 40-47 859 (2018).

860 38. Ranasinghe, R. \& Eri, R. Modulation of the CCR6-CCl20 axis: A potential 861 therapeutic target in inflammation and cancer. Med. 54, (2018). 
862 39. Rubie, C. et al. CCL20/CCR6 expression profile in pancreatic cancer. J. Transl.

863 Med. 8, 1-8 (2010).

864 40. Osuala, K. O. \& Sloane, B. F. Many Roles of CCL20: Emphasis on Breast Cancer.

865 Postdoc J. 2, 7-16 (2014).

866 41. Kindermann, R. \& Snell, J. L. Markov Random Fields and their applications.

867 (American Mathematical Society, 1980). doi:http://dx.doi.org/10.1090/conm/001

868 42. Haribhai, D. et al. Regulatory T cells dynamically control the primary immune

869 response to foreign antigen. J. Immunol. 178, 2961-2972 (2007).

870

$\$ 71$

$\$ 72$

$\$ 73$

$\$ 74$

$\$ 75$

$\$ 76$

$\$ 77$

$\$ 78$

$\$ 79$

$\$ 80$

$\$ 81$

$\$ 82$ 


\section{FIGURES}

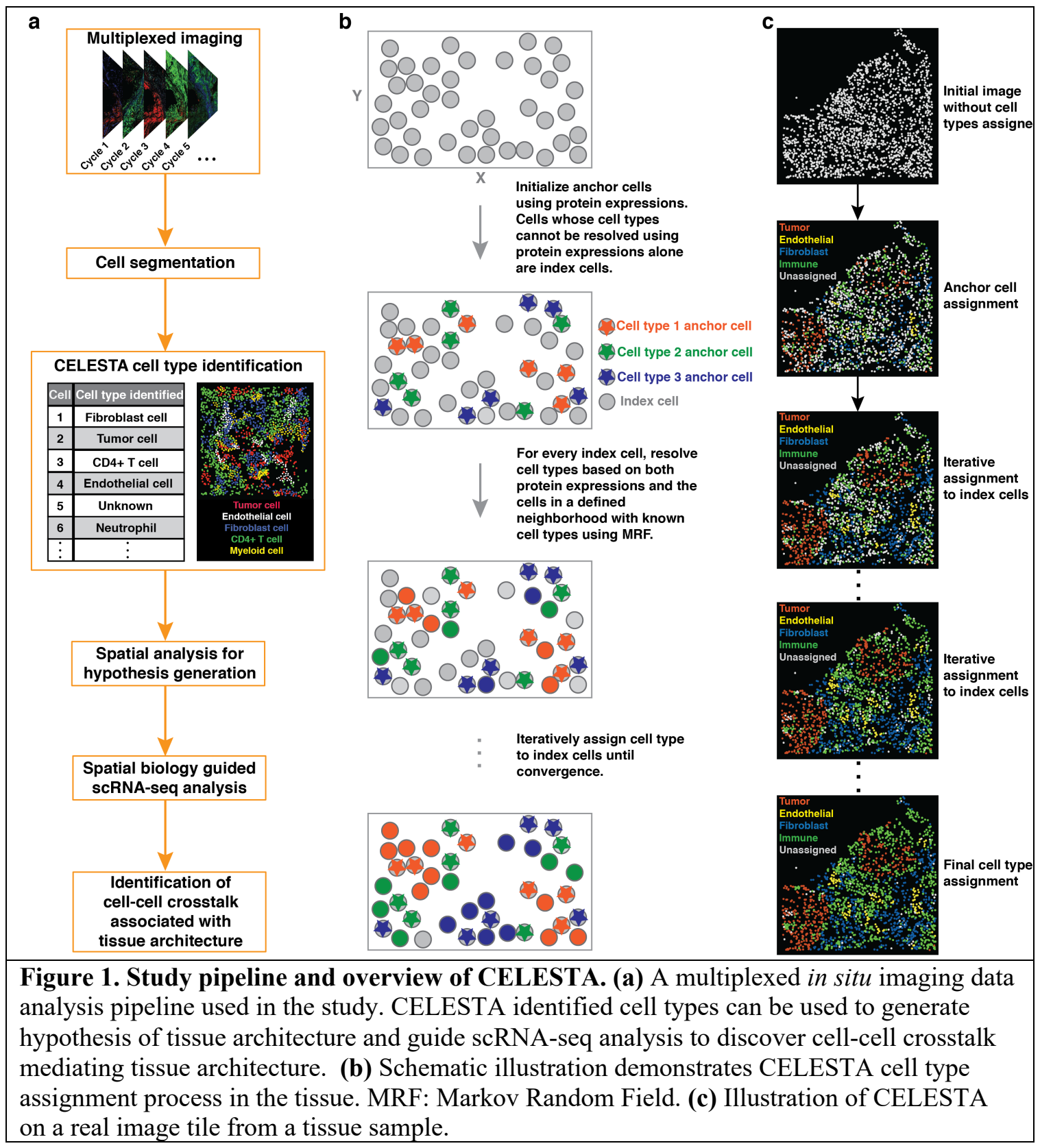




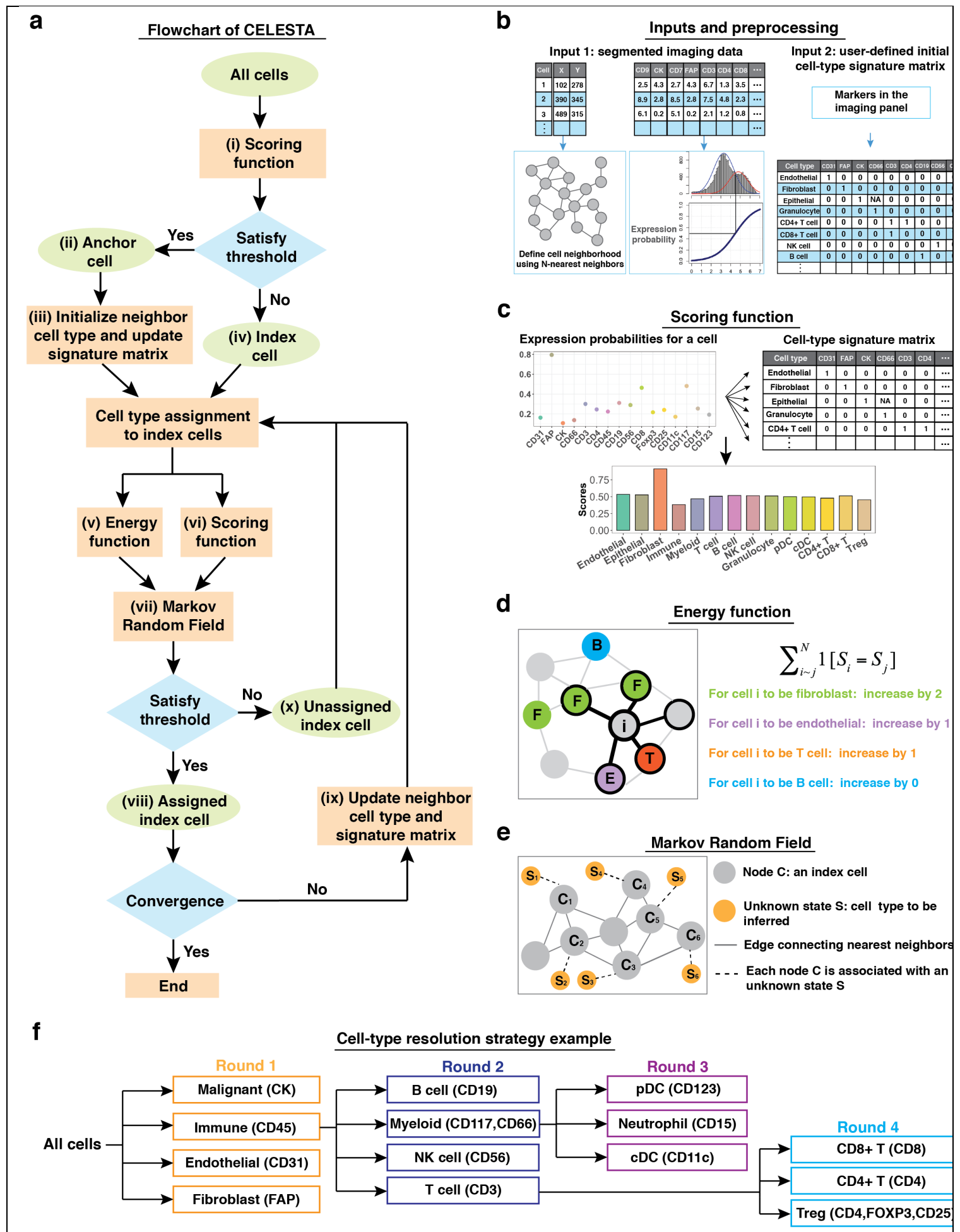

Figure 2. Illustration of key steps in CELESTA. (a) Flowchart of CELESTA algorithm. (b) CELESTA inputs and data processing. (c) An example illustrates CELESTA scoring unction. pDC: plasmacytoid dendritic cell. cDC: conventional dendritic cell. CK: cytokeratin. (d) An example illustrates neighborhood of an index cell $i$. The cell type information from spatial nearest neighboring cells of index cell $i$ is added to the field by an energy function using Potts model indicator function. (e) We assume that each index cell $C$ is associated with an unknown 
bioRxiv preprint doi: https://doi.org/10.1101/2022.02.02.478888; this version posted February 5, 2022. The copyright holder for this preprint

(which was not certified by peer review) is the author/funder. All rights reserved. No reuse allowed without permission.

state $S$ which is the cell type to be inferred. Cells are represented as nodes in an undirected graph with edges connecting the neighboring cells. We assume that the joint distribution of $S$ 's satisfies discrete Markov Random Field. (f) An example demonstrating cell-type resolution strategy based on our HNSCC imaging panel.

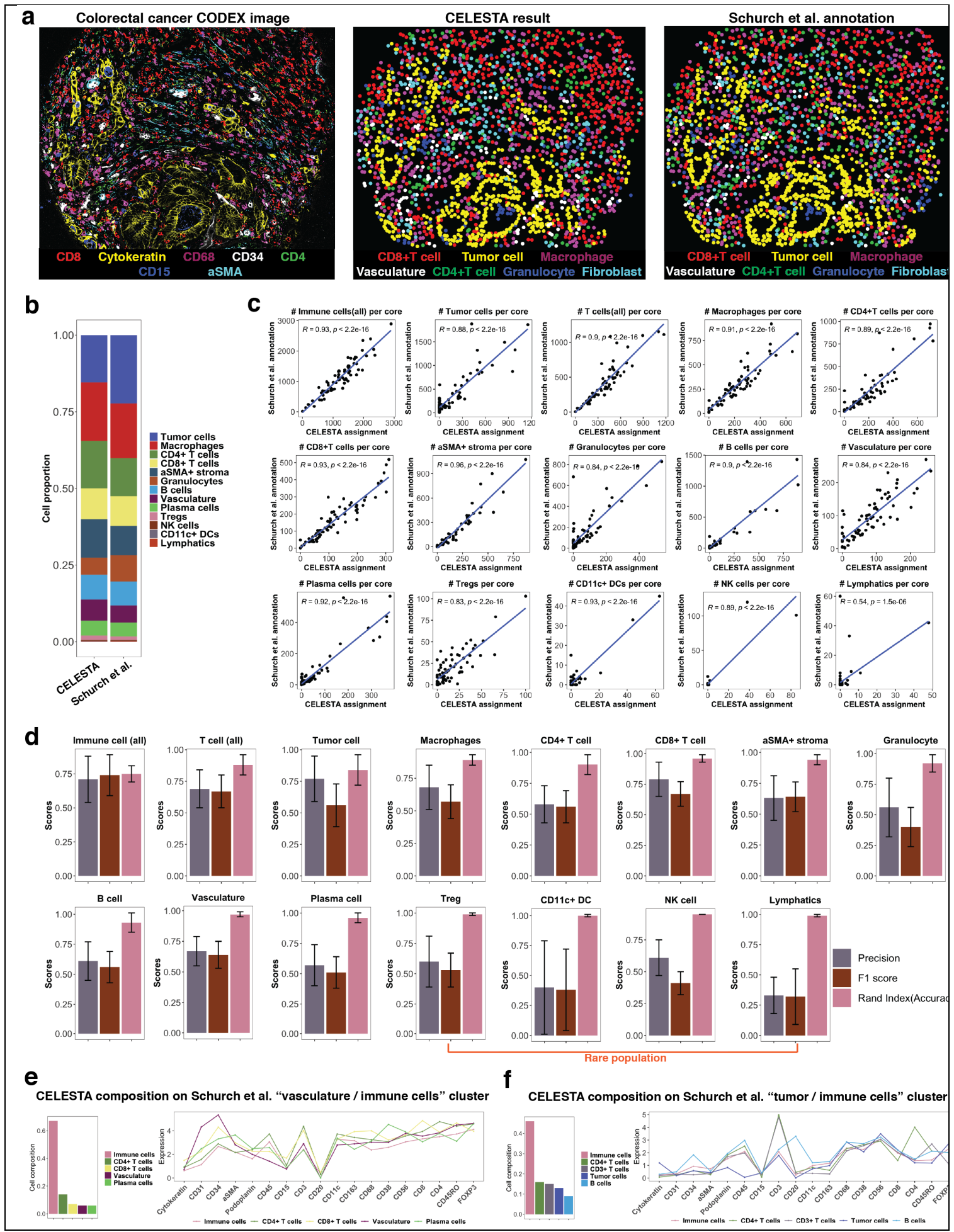




Figure 3. CELESTA applied to a public CODEX dataset of colorectal cancer tissue
microarray (Schurch et al.). (a) An example tissue microarray (TMA) core region of
CODEX image with seven channel overlay (Left), image derived from CELESTA assigned
cell types (Middle) and image derived from Schurch et al. annotated cell types (Right). (b) Cell
type compositions from CELESTA inferred cell types and Schurch et al. annotations. (c)
Correlation diagrams show number of cells identified per TMA core across 70 core regions
between CELESTA and Schurch et al. annotations for each cell type. Numbers of cells
identified per TMA core region for each cell type are correlated between CELESTA identified
cell types and Schurch et al. annotations. (d) Precision scores, F1 scores, and accuracy (Rand
Index) scores for major cell types identified by CELESTA using Schurch et al. annotations as
"ground truth". Error bars are calculated using standard deviations across 70 TMA core regions
in the data. Rare population is defined as average of fewer than 20 cells per core region in the
annotations. (e) CELESTA cell type assignments on a cluster of cells which Schurch et al.
annotated as a mixture of vasculature or immune cells. CELESTA cell type compositions are
shown on the left panel and average canonical marker expressions for each cell type in the
cluster are shown on the right panel. (f) CELESTA cell type assignments on a cluster of cells
which Schurch et al. annotated as a mixture of tumor or immune cells. CELESTA cell type
compositions are shown on the left panel and average canonical marker expressions for each
cell type in the cluster are shown on the right panel.




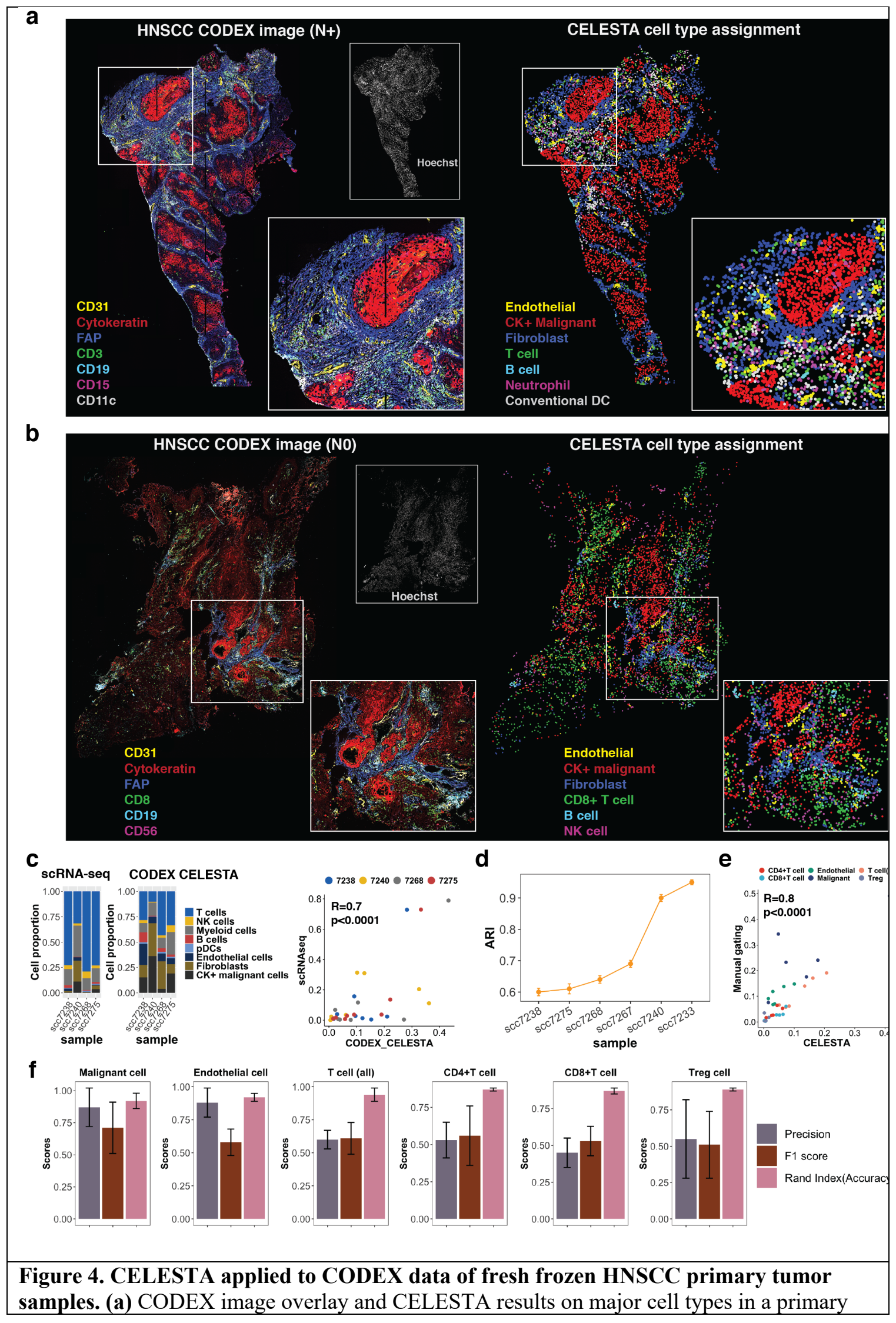


tumor tissue sample of head and neck squamous cell carcinoma (HNSCC) with lymph node metastasis $(\mathrm{N}+)$. CK: Cytokeratin. (b) CODEX image overlay and CELESTA results on major cell types in a primary tumor tissue sample of HNSCC without lymph node metastasis (N0). (c) Cell type compositions from scRNA-seq data (Left) and CELESTA inferred cell types on CODEX data (Middle). scRNA-seq data were obtained from proximal tissue slices with the CODEX data tissue on the same four patient samples. Correlation between CELESTA inferred cell compositions and scRNA-seq cell compositions on the same four samples (Right). (d) Adjusted rand index (ARI) to assess CELESTA's performance against manual gating for each tissue sample. Error bars were calculated based on 50 runs of random sampling. (e) Correlation between CELESTA inferred cell compositions and manual gating compositions. (f) Cell type precision scores, F1 scores and accuracy scores (Rand index) on cell types relevant to downstream analysis (malignant cells, endothelial cells and T cell subtypes). 


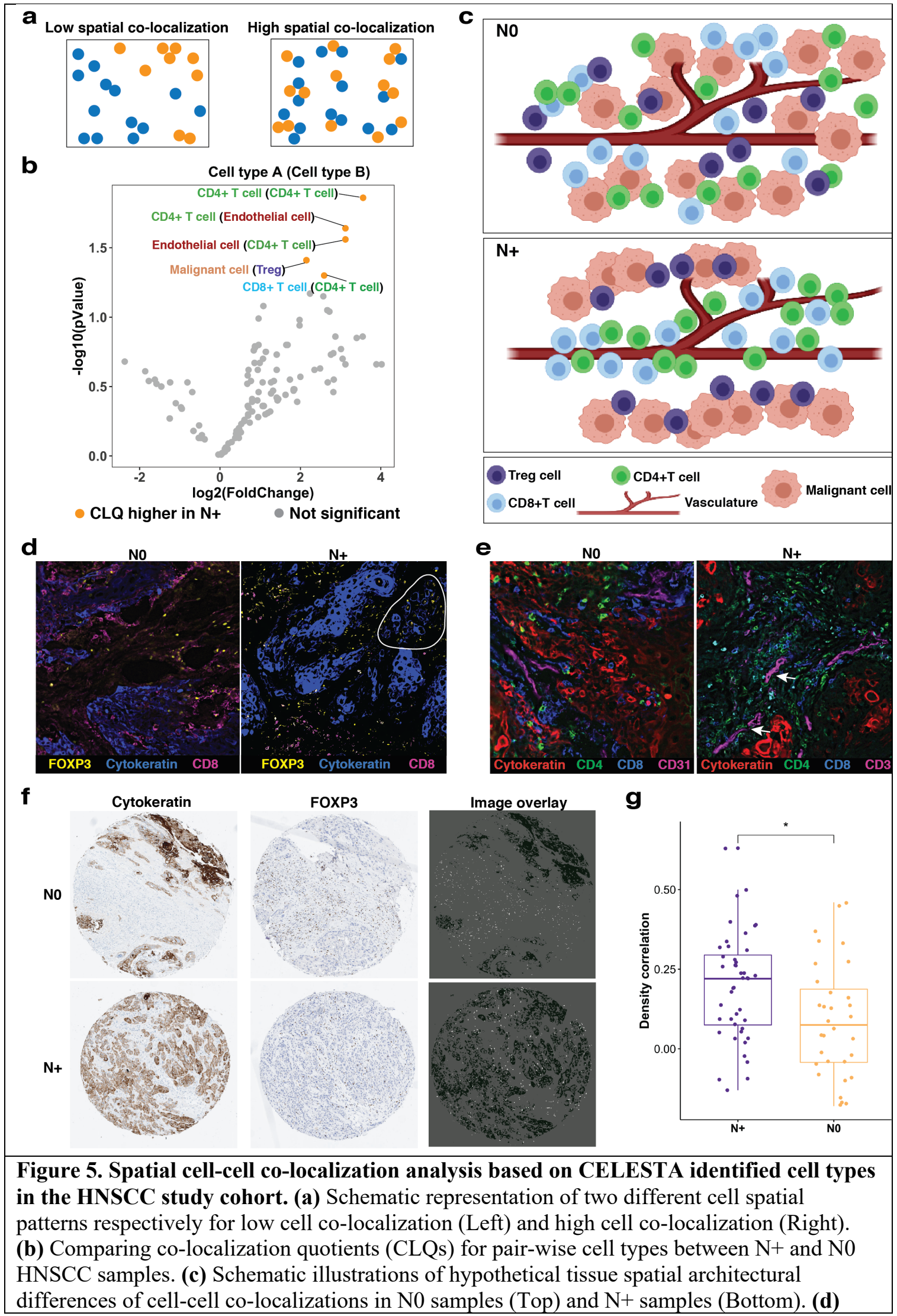


Representative regions for N0 sample (Left) and N+ sample (Right) depicted as three-color overlay images with FOXP3 (yellow), Cytokeratin (blue) and CD8 (magenta). (e)

Representative regions for N0 sample (Left) and N+ sample (Right) depicted as four-color overlay images with Cytokeratin (red), CD4 (green), CD8 (blue) and CD31 (magenta). (f) Representative HNSCC TMA cores for N0 and N+ patients depicted as overlay images with Cytokeratin and FOXP3 staining. (g) Density correlation analysis shows that Cytokeratin and Foxp3 have higher density correlations in $\mathrm{N}+$ patients $(\mathrm{n}=44)$ than $\mathrm{N} 0$ patients $(\mathrm{n}=32)$ in an independent TMA cohort of HNSCC samples. *p-value $<0.05$ 


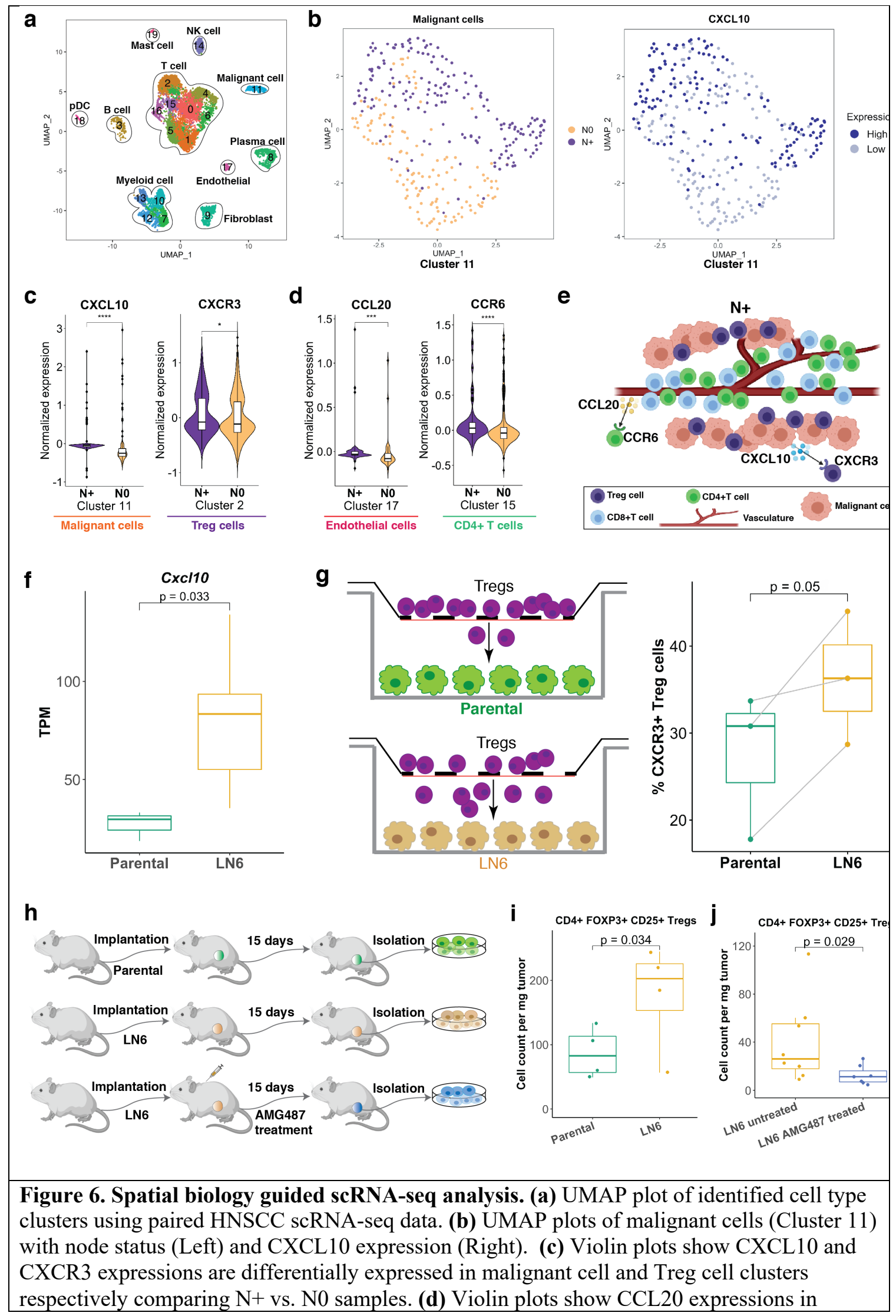


endothelial cell cluster and CCR6 expressions in CD4+T cell cluster between $\mathrm{N}+$ and $\mathrm{N} 0$ samples. (e) Schematic illustration shows cell-cell crosstalk with identified chemokine ligandreceptor pairs mediating cellular spatial co-localization in $\mathrm{N}+$ samples. (f) Cxcl10 expression is significantly higher in mouse model LN6 generation cell lines than parental cell lines. (g) Transwell experiment shows that LN6 malignant cells attract more CXCR3+ Treg cells through the membrane than parental cell lines. (h) Schematic workflow of in vivo experiments. (i) LN6 line tumors recruit more Treg cells into the tumors than parental tumors. (j) AMG487 treatment significantly reduces Tregs recruited into the LN6 tumors. *: adjusted p-value $<$ $0.05, * *$ : adjusted $\mathrm{p}$-value $<0.01, * * *$ : adjusted $\mathrm{p}$-value $<0.005, * * * *$ : adjusted $\mathrm{p}$-value $<$ 0.001 . 